\title{
Synthese und Reaktionen von Trisdimethylaminomethan
}

Von Prof. Dr. H. Bredereck, Dr. F. Effenberger und Dipl.-Chem. Th. Brendle

Institut für Organische Chemie der Technischen Hochschule Stuttgart

Es ist uns gelungen, als ersten Vertreter der Trisdialkylaminomethane das Trisdimethylaminomethan (1) durch Umsetzung von Tetramethylformamidinium-Salzen [1] mit Alkalimetalldimethylamiden in guter Ausbeute darzustellen.

$$
\begin{gathered}
{\left[\left(\mathrm{CH}_{3}\right)_{2} \mathrm{~N}-\mathrm{CH}=\mathrm{N}\left(\mathrm{CH}_{3}\right)_{2}\right]^{\oplus} \mathrm{X}^{\ominus}+\mathrm{M}-\mathrm{N}\left(\mathrm{CH}_{3}\right)_{2} \rightarrow} \\
\left(\mathrm{CH}_{3}\right)_{2} \mathrm{~N}-\mathrm{HC}^{\mathrm{N}\left(\mathrm{CH}_{3}\right)_{2}}+\mathrm{MX} \\
\mathrm{N}\left(\mathrm{CH}_{3}\right)_{2} \\
\mathrm{X}=\mathrm{Cl}, \mathrm{ClO}_{4} \quad \mathrm{M}=\mathrm{Li}, \mathrm{Na}, \mathrm{K}
\end{gathered}
$$

(1) reagiert glatt mit $\mathrm{CH}$-aciden Verbindungen zu Dimethylaminomethylen-Verbindungen (2) ${ }^{[2]}$ und mit $\mathrm{NH}_{2}$-Verbindungen zu Amidinen (3) [3].

$$
\begin{aligned}
& \text { (1) }+\mathrm{H}_{2} \mathrm{C}_{\mathrm{Y}}^{\mathrm{X}} \rightarrow\left(\mathrm{CH}_{3}\right)_{2} \mathrm{~N}-\mathrm{CH}=\mathrm{C}_{\mathrm{Y}}^{\mathrm{X}}+2\left(\mathrm{CH}_{3}\right)_{2} \mathrm{NH} \\
& \text { (2) } \\
& \begin{array}{c|c|c|c}
\mathrm{X} & \mathrm{CN} & \mathrm{COOCH}_{3} & \mathrm{C}_{6} \mathrm{H}_{5} \mathrm{CO} \\
\hline \mathrm{Y} & \mathrm{CN} & \mathrm{CN} & \mathrm{H}
\end{array} \\
& (1)+\mathrm{RNH}_{2} \rightarrow\left(\mathrm{CH}_{3}\right)_{2} \mathrm{~N}-\mathrm{CH}=\mathrm{NR}+2\left(\mathrm{CH}_{3}\right)_{2} \mathrm{NH}
\end{aligned}
$$


Synthesebeispiele:

Trisdimethylaminomethan: $\mathrm{Zu}$ einer Aufschlämmung von Lithiumdimethylamid, dargestellt aus $15,2 \mathrm{~g}$ Lithium, $137 \mathrm{~g}$ $\mathrm{n}$-Butylbromid und $180 \mathrm{~g}$ Dimethylamin nach ${ }^{[4,5]}$, in $600 \mathrm{ml}$ wasserfreiem Äther gibt man bei $-20^{\circ} \mathrm{C} 68 \mathrm{~g}$ Tetramethylformamidinium-chlorid. Anschließend leitet man durch die Apparatur Stickstoff und rührt $15 \mathrm{Std}$. bei Raumtemperatur. Nach Abdestillieren des Äthers wird im Vakuum (Wasserstrahlpumpe) das Trisdimethylaminomethan abdestilliert. Rohausbeute: $51,0 \mathrm{~g}, \mathrm{Kp}=40-43^{\circ} \mathrm{C} / 12$ Torr. Nach Destillation über eine Einstichkolonne: Reinausbeute $46,0 \mathrm{~g}$ $(63 \%), \mathrm{Kp}=42-43^{\circ} \mathrm{C} / 12$ Torr.

$\alpha$-Cyan- $\beta$-dimethylaminoacrylsäure-methylester: $Z_{u l} \quad 2,48 \mathrm{~g}$ Cyanessigsäuremethylester in $50 \mathrm{ml}$ wasserfreiem Äther läßt man bei Raumtemperatur unter Rühren und Feuchtigkeitsausschluß 3,63 $\mathrm{g}$ Trisdimethylaminomethan in $50 \mathrm{ml}$ wasserfreiem Äther zutropfen, erhitzt 15 min unter Rückfluß und saugt ab. Rohausbeute: $3,8 \mathrm{~g}, \mathrm{Fp}==97-100^{\circ} \mathrm{C}$, nach $\mathrm{Um}$ kristallisieren aus $30 \mathrm{ml}$ Aceton/Petroläther (1:1) Reinausbeute: $3,2 \mathrm{~g}(83 \%), \mathrm{Fp}=101-102{ }^{\circ} \mathrm{C}$.
$\mathbf{N}, \mathbf{N}$-Dimethyl- $\mathbf{N}^{\prime}$-p nitrophenylformamidin: Aus 3,45 g $\mathrm{p}$ Nitroanilin in $50 \mathrm{ml}$ wasserfreiem Äther und $3,63 \mathrm{~g}$ Trisdimethylaminomethan in $50 \mathrm{ml}$ wasserfreiem Äther wie vorstehend beschrieben. Nach dem Erhitzen wird jedoch der Äther am Rotationsverdampfer abdestilliert, und die gelbgrünen Kristalle $\left(4,8 \mathrm{~g}, \mathrm{Fp}=70-78^{\circ} \mathrm{C}\right)$ werden aus $40 \mathrm{ml}$ wasserfreiem Äthanol umkristallisiert, Ausbeute: 4,2 g $(87 \%), F p=82-83^{\circ} \mathrm{C}$.

Eingegangen am 25. Oktober 1965

[Z 95]

[1] Z. Arnold, Coll. czechoslov. chem. Commun. 24, 760 (1959) [2] H. Meerwein, W. Florian, N. Schön u. G. Stopp, Liebigs Ann. Chem. 641, 1 (1961).

[3] H. Bredereck, F. Effenberger u. A. Hofmann, Chem. Ber. 97, 61 (1964).

[4] C, Weygand u. G. Hilgetag: Organisch-chemische Experimentierkunst. Barth-Verlag, Leipzig 1964, S. 745.

[5] Houben-Weyl: Methoden der Organischen Chemie. ThiemeVerlag, Stuttgart 1958, Bd. 2/3, S. 184. 